\title{
Habitat saturation, benefits of philopatry, relatedness, and the extent of co-operative breeding in a cichlid
}

\author{
Dik Heg, Susan Rothenberger, and Roger Schürch \\ Department of Behavioural Ecology, Institute of Ecology and Evolution, University of Bern, \\ Wohlenstrasse 50a, CH-3032 Hinterkappelen, Switzerland
}

Co-operative breeding in vertebrates may emerge due to subordinates delaying dispersal when free breeding habitat is not available ('habitat saturation' hypothesis, HS). However, delayed dispersal might also be due to younger individuals postponing dispersal to when they are more competitively able or have more to gain from breeding independently ("benefits-of-philopatry" hypothesis, BP) or to when inclusive fitness benefits no longer outweigh the benefits from independent breeding ("kin selection" hypothesis, KS). Here, we show in three experiments that both HS and BP determine the extent of co-operative breeding in the cichlid Neolamprologus pulcher. Contrary to the KS, individuals significantly avoided settlement with related individuals, and an additional settlement experiment confirmed this result. Our results suggest that kin structure in these cichlids emerges from limits on dispersal, but if such barriers are absent, cichlids prefer to settle with unrelated individuals to maximize the benefits of direct reproductive participation. Key words: body size, cichlidae, co-operation, density-dependence, helper, status. [Behav Ecol 22:82-92 (2011)]

$\mathrm{T}$ he habitat saturation hypothesis (HS) has been invoked to explain the extent of co-operative breeding in vertebrate societies (Selander 1964). This hypothesis states that offspring delay dispersal and may help instead because free habitat to settle, and breed in, is not available. The effect may be more pronounced if, for instance, predation risk makes dispersal less worthwhile or more risky ("ecological constraints" Emlen 1982; Heg et al. 2004a) or when the benefits of staying at home are high (Stacey and Ligon 1991; e.g., van Vuren and Armitage 1994; Tibbetts and Reeve 2003; Griesser et al. 2006; Ridley and Raihani 2007) e.g., through kin-selected benefits (Grafen 2007). The HS has been tested by experimental removal of dominants from groups (Koenig and Dickinson 2004; Stiver et al. 2006), population density manipulations (Komdeur 1992; Harris et al. 1995; Lucia et al. 2008), and provisioning of extra breeding sites (Walters et al. 1992; Bull and Schwarz 1996; Langer et al. 2004; Heg et al. 2004a; Bergmüller et al. 2005). However, the general applicability of the habitat saturation hypothesis has been challenged, as some populations also show extensive co-operative breeding in the presence of suitable and free surrounding habitat for subordinates to disperse to and breed independently (Macedo and Bianchi 1997; Baglione et al. 2005; Heg et al. 2008a).

As an alternative to the HS, the benefits-of-philopatry hypothesis (BP) has been invoked to explain why subordinates delay dispersal (Pen and Weissing 2000; Cahan et al. 2002; Covas and Griesser 2007). This hypothesis states that subordinates strategically delay dispersal because the life-time benefits of doing so outweigh those from immediately attempting dispersal. It is particularly likely to apply in fish (e.g., cooperatively breeding cichlids Heg et al. 2006), which show indeterminate growth, where younger smaller cichlids stand

\footnotetext{
Address correspondence to D. Heg. E-mail: dik.heg@iee.unibe.ch.

Received 7 December 2009; revised 23 September 2010; accepted 23 September 2010.
}

(C) The Author 2010. Published by Oxford University Press on behalf of the International Society for Behavioral Ecology. All rights reserved.

For permissions, please e-mail: journals.permissions@oup.com a substantially higher risk of predation compared with older larger cichlids, and small cichlids may gain protection from living in groups (Heg et al. 2004a). Younger cichlids are also more related to the recipients of their helping behavior than older cichlids (Dierkes et al. 2005; Stiver et al. 2005), so kin selection may also explain delayed dispersal in these cichlids.

Neolamprologus pulcher lives in co-operatively breeding groups consisting of a dominant breeding pair and up to 14 subordinate helpers (Balshine et al. 2001) and is endemic to Lake Tanganyika. Offspring delay dispersal and remain as subordinate helpers inside their natal group, with little emigration occurring (Stiver et al. 2007). Frequent breeder exchanges results in genetic relatedness of the subordinates to the breeders progressively declining with helper age (Dierkes et al. 2005). Experiments have shown that ecological constraints may affect the incidence of subordinate dispersal and independent breeding (Heg et al. 2004a; Bergmüller et al. 2005). All group-living cichlids show distinct size hierarchies, partly maintained by status and strategic adjustments in growth (Heg et al. 2004b; Hamilton and Heg 2008). The largest group members form the dominant breeding pair, and subordinates are reproductively suppressed (Fitzpatrick et al. 2006; Heg 2008), subordinate reproduction being rare both in the laboratory (Heg et al. 2006; Heg and Hamilton 2008; Heg 2008; Heg et al. 2009) and in the field (Stiver et al. 2009). Neolamprologus pulcher is a substrate-spawning cichlid attaching its eggs secluded under rocks and in crevices. Under laboratory conditions, it can be induced to use flowerpot halves for spawning (Taborsky 1984) and to breed in tightly packed groups (Limberger 1983).

Here, we show experimentally that small cichlids from the co-operatively breeding species, $N$. pulcher, prefer to settle as subordinates with nonkin, notwithstanding they are reproductively capable, the habitat is not saturated and predators are not present. In the first experiment, we test the hypothesis that small females might delay dispersal because they are not reproductively capable or less capable compared with later in life when they have grown larger and to full maturity. We compare the clutch sizes and average egg masses of females growing from a small to a large size (repeated measures of 
individual females). If small females do not reproduce, despite they are dominant and therefore not reproductively suppressed (Heg 2008), they might derive more benefits from philopatry than dispersal due to protection inside their group (e.g., Heg et al. 2004a) and delay dispersal until they are reproductively capable. If small subordinate females are unlikely to reproduce because they are subdominant in groups compared with similar-sized dominant females (see also Heg 2008), reproductive benefits from philopatry are unlikely to apply, and they could derive more reproductive benefits from dispersal and establish a new group as a dominant female.

In the second experiment, we test the hypothesis that both small males and small females delay dispersal because they cannot successfully compete with larger conspecific competitors for free habitat if an empty breeding patch is provided and instead opt for a subordinate position. We test this hypothesis by releasing 5 differently sized males and females into a large tank with 1 empty breeding patch available and expected the largest male and female to gain the dominant breeding positions and the smallest males and females to be accepted as subordinates.

In the third experiment, we test the hypothesis that subordinates delay dispersal because the availability of suitable free habitat is limited (the habitat saturation hypothesis). We induce habitat saturation by sequentially releasing 3 males and 3 females of 3 different size classes inside a large octagonal tank (ringtank) every third day, and they were allowed to settle in any of the 24 breeding patches. This was repeated 12 times, so the population increased from 6 to 72 fish. If the HS applies, all fish should attempt to pair up and breed at initial low densities. With increasing density, only the smaller fish should settle as subordinates, whereas larger fish should be competitively excluded. If the BP applies, smaller fish should attempt to settle as subordinates from the very start, even when free breeding habitat is available.

In the third experiment, we also allowed close kin to settle sequentially (e.g., a large, medium, and small sibling released on days 0,3 , and 6 , respectively): If the kin selection hypothesis applies, subordinate cichlids should prefer to settle with close kin (e.g., associate with a related dominant male or female already settled inside the ringtank, see also Jordan et al. 2010). However, sequential settlement under a competitive laboratory setting might make it difficult for cichlids to settle close to kin because their previous association has been disrupted by us. Therefore, we also conducted a fourth experiment, where 4 groups of 6 close kin were released simultaneously and the kin could remain together and settle together or disassociate and settle with any of the other fish. Again, if kin selection applies, the siblings are expected to remain together. In experiments 3 and 4 , we expected kin to settle with nonkin if they target own reproduction (avoid inbreeding) or avoid competition with close kin for mates.

\section{MATERIALS AND METHODS}

\section{Study animals}

All experiments were conducted with $N$. pulcher descendants, from parents originally wild caught near the village of Kasakalawe (Zambia) in 1996 and held in separate breeding stock families from that year onwards. The breeding stock has retained substantial genetic variation: The number of alleles and the levels of heterozygosity estimated using microsatellite DNA markers does not differ between our stock (Heg et al. 2006; Heg 2008) and the natural source population (Dierkes et al. 2005). Note that $N$. pulcher is able to recognize conspecifics individually (Hert 1985; Balshine-Earn and Lotem
1998), so relatives released in experiments 3 and 4 should be able to find (experiment 3) or respectively stay together (experiment 4), with their relatives if they wanted to. All individuals in all experiments were sexed by inspection of the gonadal papilla. Individuals were resexed at the end of the experiments when most individuals had reached mature size (>30 mm standard length [SL]), and sexing was more reliable. In experiments 1 and 2, all individuals were correctly sexed, and in experiments 3 and 4, missexing of some individuals resulted in slight alterations in the final number of males and females released (see Table 1).

\section{Experiment 1: body size, status, and reproduction}

Reproduction of dominant and subordinate females was recorded in 94 groups for an average period of 226 days (range 25-675 days) in tanks of 50-100 1 in climate-controlled rooms (study period 23 March 2006 to 2 February 2008). This period encompassed the estimated ages of dominant females in nature (Balshine et al. 2001; Heg et al. 2005: see area between hatched lines in Figure 2a) and females in the laboratory produce on average 1 clutch every 14 days (e.g., Heg 2008). All group members $>20 \mathrm{~mm}$ SL were individually marked with fin clips and remarked and measured (body mass in milligrams and body size standard length SL in millimeter) every 30 days. To test whether small dominant females are reproductively capable, the 94 groups included 18 pairs newly formed from a small female and a small male (at the start of the experiment between 28.5 and $42.0 \mathrm{~mm}$ SL, females always smaller than the males). Broods were assigned to dominant and subordinate females based on prespawning behavior (cleaning of the pots, courting, and pseudo-spawning with the dominant male, which they do very intensively in the prespawning period, i.e., usually every minute and at least every 5 min) 1-2 days before spawning and actual spawning behavior by daily observations. This method has been proved to be extremely reliable in determining the mother of a brood (Heg et al. 2008b). Tanks were fed daily with Tetramin cichlid flake food. In total, 78 dominant females produced on average 7.0 broods (range 1-22), and their average body size was $59.4 \mathrm{~mm}$ SL (range 35.3-80.3 mm). Dominant females who failed to produce broods were replaced with a new dominant female from 400-l tank containing aggregation fish, as this was typical to occur in newly established pairs. In total, 13 subordinate females from 13 different groups produced on average 2.4 broods (range 1-11), and their average body size was $48.9 \mathrm{~mm}$ SL (range $32.0-60.4 \mathrm{~mm}$ ). Note that in 6 of the groups where a subordinate female reproduced, the dominant female did not produce any clutches, although she had produced clutches before the experiment started.

Clutch sizes were determined in the evening and an average egg mass was determined after a sample of eggs per brood were dried for $32 \mathrm{~h}$ in a stove at $70^{\circ} \mathrm{C}$. Groups contained between 0 and 30 subordinates that were larger than $25 \mathrm{~mm}$ SL. Group sizes changed during the experimental period due to the growth of offspring who were recruited as subordinates and due to expulsion of subordinates, who were removed from the tank (average change in the number of subordinates \pm standard deviation [SD], dominants with broods: $2.8 \pm 6.2$, range: $0-30, n=78$; subordinates with broods: $0.23 \pm 0.44$, range: $0-1, n=13$ ). Therefore, actual number of subordinates present during reproduction were as follows: groups with dominant female broods-average \pm SD: $2.0 \pm 3.6$, range: $0-23, n=78$ and groups with subordinate female broods-average $\pm \mathrm{SD}$ : $3.4 \pm 2.5$, range: $1-9, n=13$. Ages of female dominants and subordinates fish at the time of each clutch production were estimated using the Blumberg's hyperlogistic growth curve equation published elsewhere, i.e., intrapolating their size SL at clutch production 
Table 1

Experiments 2-4: body sizes of females and males in the trials

\begin{tabular}{|c|c|c|c|c|c|}
\hline \multirow{2}{*}{$\begin{array}{l}\text { Sex } \\
\text { Size class }\end{array}$} & \multicolumn{5}{|c|}{ Average body sizes \pm SD (range) per size class } \\
\hline & 1 & 2 & 3 & 4 & 5 \\
\hline \multicolumn{6}{|c|}{ Experiment $2(n=20$ each cell $)$} \\
\hline Males & $37.7 \pm 1.6(35.0-40.0)$ & $42.5 \pm 1.5(40.5-45.0)$ & $47.5 \pm 1.2(45.5-50.0)$ & $52.2 \pm 1.2(50.5-55.0)$ & $57.2 \pm 1.1(55.5-60.0)$ \\
\hline Experiment 3 & Small & Medium & Large & & \\
\hline Females & $\begin{array}{l}30.6 \pm 4.9(20.3-42.0) \\
n=36\end{array}$ & $\begin{array}{l}40.4 \pm 3.6(33.2-46.0) \\
n=39\end{array}$ & $\begin{array}{l}53.9 \pm 4.6(48.0-66.2) \\
n=33\end{array}$ & & \\
\hline Experiment 4 & Small & Medium & Large & & \\
\hline Females & $\begin{array}{l}30.2 \pm 3.6(25.0-39.5) \\
n=25\end{array}$ & $\begin{array}{l}37.8 \pm 3.4(31.3-44.8) \\
n=29\end{array}$ & $\begin{array}{l}45.2 \pm 3.9(38.2-54.2) \\
n=25\end{array}$ & & \\
\hline Males & $\begin{array}{l}29.9 \pm 2.9(25.3-38.3) \\
n=23\end{array}$ & $\begin{array}{l}38.1 \pm 3.4(32.5-45.0) \\
n=19\end{array}$ & $\begin{array}{l}47.9 \pm 5.2(40.2-57.4) \\
n=23\end{array}$ & & \\
\hline
\end{tabular}

Note that the body sizes overlapped between the size classes in experiments 3 and 4 because slightly larger or smaller fish per size class were used for each trial, depending on the availability of related fish.

from the monthly body size measurements and using SL to estimate their age (Skubic et al. 2004).

\section{Experiment 2: competition for breeding positions}

This experiment was conducted from 17 April to 11 June 2006 using 3 different 400-l tanks kept in climate-controlled rooms. The experiment was designed to test how body size affects competition for dominant breeding positions and whether small fish get accepted as subordinates instead. Tanks were fed daily with Tetramin cichlid flake food. Five males and 5 females of different sizes were released simultaneously inside a 400-l tank and allowed to compete for group membership in a single breeding patch containing 2 flowerpot halves for spawning ( $n=20$ trials, each fish was individually marked, see Table 1 for the body sizes). Fish were all unrelated. Trials ended on average after 7.3 days $( \pm 3.8 \mathrm{SD}$, range $2-16$ days) when the first brood was spawned by the pair gaining the dominant breeding position (so called "dominants"), and on this day, group membership was determined for all other fish (accepted as "subordinate" or not accepted and staying in the upper half of the tank as "aggregation"). Fish were removed, and a new trial was initiated until a total sample size of 20 trials was reached; thus in total, 20 trials $\times 5$ size classes $\times 2$ sexes $=200$ different individuals were tested.

\section{Experiment 3: habitat saturation and sequential settlement with kin}

This experiment was conducted from 28 March to 14 September 2007. Cichlids were individually marked with fin clips taken from the dorsal and anal fin, individual body measurements taken (body size SL in $0.1 \mathrm{~mm}$ and body mass in $1 \mathrm{mg}$ accuracy) and sexed.

Per trial ( $n=3$ trials), 72 fish were released in sets $(n=12$ sets) of 1 large male, 1 large female, 1 medium male, 1 medium female, 1 small male, and 1 small female every third day into a large octagonal ringtank $(72001,60 \mathrm{~cm}$ height, $50 \mathrm{~cm}$ water column, and $3 \mathrm{~cm}$ sand layer of $1 \mathrm{~mm}$ grain size). Twelve flowerpot halves ( 3 large, 5 medium, and 4 small pots) and 2 stone slabs were placed within each of the 24 breeding patches as breeding substrate (see Figure 1 and Supplementary material Figure S1, S2). Cichlid food flakes Tetramin were provided through 4 automated feeders, supplemented daily with Artemia spp. eggs. The water current distributed the food through the whole tank ensuring feeding locations could not be monopolized (see Supplementary material Table S1).

Water temperature (mean $\pm \mathrm{SD}: 27.2 \pm 1.5^{\circ} \mathrm{C}$ ) and illumination cycle (lights on from 08:00 to 21:00 h) were kept constant in a climate-controlled room. The location (patches and pots visited), status (dominant, subordinate, or aggregation), and group membership (pairs and the identity of the dominants assisted by the subordinates) were determined for each individuals daily from day 0 (first set released) to day 39 (6 days after the last, 12th set was released). We also recorded the location and counted the number of aggressive behaviors received (from all other individuals already present in the ringtank) during a 5-min observation directly after release for each released individual fish separately. On day 40, all individuals were removed from the ringtank and sexed, and body measurements were taken to determine growth. This whole procedure was repeated 3 times $(n=3$ trials). Note that the locations of the breeding patches were slightly different between trial 1 , trial 2, and trial 3 . In total, 3 trials $\times$ 12 sets $\times 6$ fish $=216$ different individuals were tested.

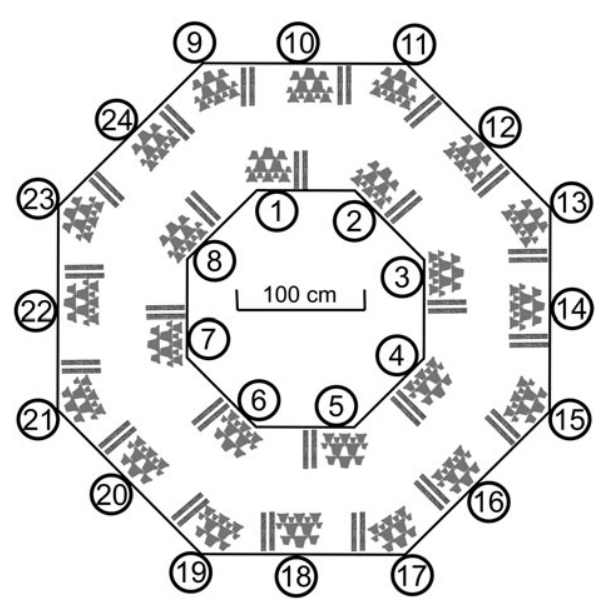

Figure 1

Setup of experiment 3. Top view of the ringtank during trial 1 , showing the 24 breeding patches, each patch contained 12 flowerpot halves and 2 stone slabs used as spawning substrate. See the Supplementary material for the setup of trials 2 and 3 . 
To test whether individuals settled preferably with related individuals, trios $(n=40$ trios $)$ or duos $(n=5$ duos $)$ of unequally sized relatives were released in different sets (e.g., large individual on day $i$, medium individual on day $i+3$, and small individual on day $i+6$ ). Note that relatives of unequal sizes were released, which avoids that kin evicted similarsized kin from their patches (e.g., subordinates matching the size of their dominants are more likely to get evicted: Balshine-Earn et al. 1998), so we reduced the likelihood that kin competition would affect our results and we increased the likelihood that relatives would settle together as well. Sexes varied randomly. These relatives were subordinates from established family groups in 50-1 tanks, and because reproduction by subordinates is rare in such small tanks, we assume that all relatives were full siblings $(r=0.5)$. Only pairwise comparisons of individuals who had successfully settled in any patch on day 39 entered the analyses (i.e., dominants and subordinates). We calculated pairwise the expected and observed numbers of patches shared. Pairwise expected patch sharing was determined using the hypergeometric distribution, based on the actual number of patches occupied by individual $i$ and the actual number of patches occupied by relative $j$ for each potential $i-j$ combination.

\section{Experiment 4: simultaneous settlement with kin}

This experiment was conducted from 7 November 2007 to 17 March 2008. Cichlids were individually marked with fin clips taken from the dorsal and anal fins, individual body measurements taken (body size SL in $0.1 \mathrm{~mm}$ and body mass in $1 \mathrm{mg}$ accuracy), and sexed. Six relatives, for each of the 4 families separately, were released simultaneously into the ringtank $(n=6$ relatives $\times 4$ families $=24$ individuals, day 0 , Table 1$)$. Relatives were from each sex and size-class combination (Table 1) and were originally subordinates from established family groups in 50-1 tanks, and because reproduction by subordinates is rare in such small tanks, we assume all relatives were full siblings $(r=0.5)$. Note that body sizes varied between trials (which was determined by the availability of relatives of particular sizes), but within each trial, body sizes were kept comparable between the families released.

The ringtank contained 4 breeding patches, where the 24 individuals could settle, form co-operative groups, and breed (see Supplementary material Figure S3). Cichlid food flakes Tetramin were provided through 4 automated feeders, supplemented daily with Artemia spp. eggs. On day 15, we determined for each individual group membership and the patches he/she occupied. Afterward, we added another 4 patches (see Supplementary material), which resulted in 1) some cichlids leaving their groups and forming new groups and 2) previously unsuccessful individuals (aggregation fish) settling. Again, on day 22, we determined for each individual, group membership and the patches he/she occupied. In total, 6 trials were conducted, which gives a total sample size of 144 individuals tested, minus 1 medium male who died shortly after the release into the ringtank. We expected the large fish to form pairs with unrelated large partners to avoid inbreeding. Under the kin selection hypothesis, we expected the medium and small cichlids to remain with their kin. Alternatively, if subordinates target their own participation in reproduction, small and medium cichlids should avoid settlement with kin.

\section{Ethical statement}

We provided plastic hiding tubes near the surface of the tanks in each experiment. These tubes were used by helpers being evicted from the group (experiment 1, evicted helpers were removed from their group) or fish failing to settle in the breed- ing shelters (experiments 2, 3, and 4; in these 3 experiments, tanks were large and fish could swim around the tank in the upper water layer and thereby avoid overt conflicts or terminate chases). Moreover, food was always provided on the water surface (and then the food started to sink slowly), so fish staying near the water surface had direct access to food. Consequently, none of the fish being evicted or failing to settle experienced a severe reduction in body condition nor signs of prolonged stress. The experiments described in this paper were approved by LANAT of the Kanton Bern, license number $40 / 05$.

\section{Data analyses}

All analyses were conducted in SPSS 16.0.

\section{Experiment 1}

Two dependent variables were analyzed using general linear mixed modeling with individual identifier nested within status as random effects (Norusis 2007): 1) clutch size (log10 transformed before analysis) and 2) average egg mass. Clutch size and average egg mass were then related to the following independent variables: female body size SL in millimeters, female status (dominant or subordinate), and number of subordinates $>25 \mathrm{~mm}$ SL inside their group (i.e., potential helpers) because they are reported to affect female reproduction (Taborsky et al. 2007). Interactions between the independent variables were also tested, but because they were nonsignificant, dropped from the models. Note that individuals did not change status during this experiment.

\section{Experiment 2}

Per trial, 10 individuals ( 5 males and 5 females) competed for access to group membership, so the dependent variable status obtained at the end of the experiment per focal fish has one of three possibilities: dominant, subordinate, or aggregation fish, which follows a multinomial distribution (Norusis 2007). Status achieved was then related to focal sex (male or female), focal body size SL in millimeters, and their interaction using Multinomial Regression with aggregation fish as the reference category (Norusis 2007). The body size difference between dominants and same-sex subordinate or same-sex aggregation fish was analyzed with an analysis of variance (ANOVA), with trial as random effect and the fixed effects of status (same-sex subordinate or same-sex aggregation fish), sex of dominant (male or female), and their interaction.

\section{Experiment 3}

The number of patches visited in the $5 \mathrm{~min}$ after release into the ringtank follows a Poisson distribution (Norusis 2007) and was analyzed using a generalized linear model with a log-link (Poisson GLM), with trial, size class (small, medium, or large), and day of release $(0,3,6 \ldots 33)$ as effects. The number of patches visited in the 5 min after release was correlated with aggression received by already established individuals using Spearman rank correlation. The daily number of dominant females or males settled inside the ringtank was analyzed with a Poisson GLM, with effects of sex, day of release, sex $\times$ day of release, and trial as effects. The number of subordinates accepted by each dominant female was analyzed with a Poisson GLM, with effects of day of release and trial. The status achieved by each focal individual at day 39 of the trials (dominant, subordinate, or aggregation fish) was analyzed with a multinomial regression, with effects of day of release, body size SL in millimeters, sex, and trial. Interactions were nonsignificant and removed from the main effects model. The expected versus observed patches shared by relatives were 


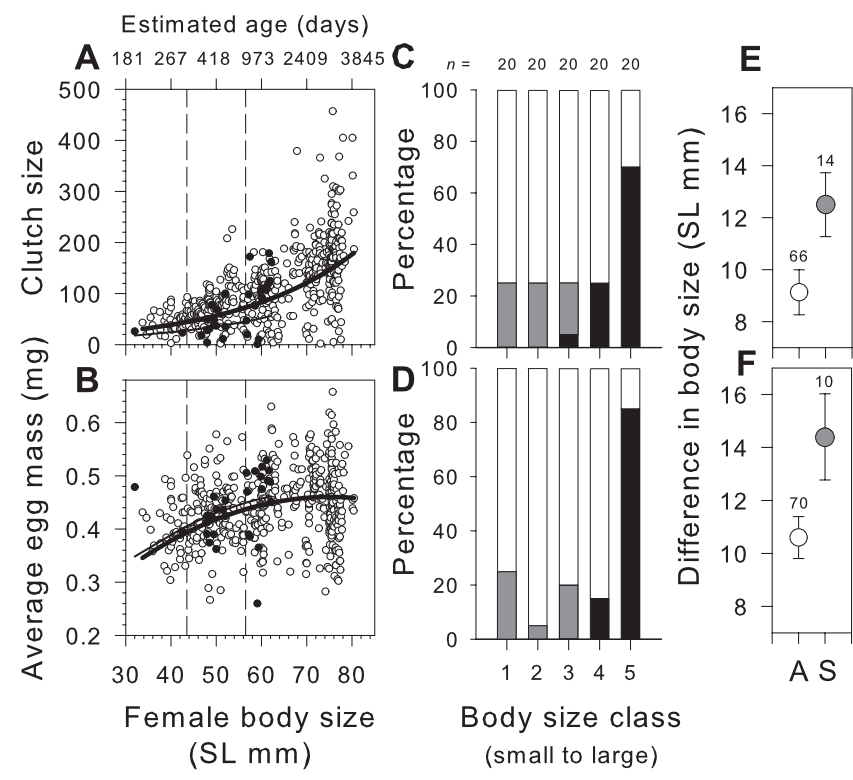

Figure 2

Experiments 2 and 3: body size, reproduction, and competitive success. Experiment 1, female reproduction: (a) clutch size and (b) average egg mass for dominant (white circles, bold line) and subordinate females (black circles, thin line). The area between the dashed lines delineates the body sizes of dominant females occurring in nature. See for statistics Table 2. Experiment 2: competition for group membership. (c) Female and (d) male likelihood of acquiring a dominant (black bars) or subordinate position (gray bars) depended on their body size (see for size classes 1 small to 5 large: Table 1) compared with remaining in the aggregation (white bars, $n=20$ trials, see for statistics Table 3) (e and f) The size difference between dominants and same-sex subordinates (gray circles) was significantly larger than those between dominants and same-sex aggregation fish who were not accepted inside their group (white circles) for both (e) females and (f) males (means \pm SEM with sample sizes depicted, see for statistics main text).

compared using Wilxocon's tests. Only 7 cichlids shared at least 1 patch with a relative (see the results and supplementary material Table S2), so no additional analyses were performed beyond those reported in the main text.

\section{Experiment 4}

Only individuals who had successfully settled in any patch on day 15 and/or day 22 entered the analyses (see the Supplementary material Table S3), that is they settled either with relatives or with nonrelatives. We calculated pairwise the expected and observed numbers of patches shared for day 15 (4 patches) and day 22 (8 patches) separately. Pairwise expected patch sharing was determined using the hypergeometric distribution, based on the actual number of patches occupied by the smaller individual $i$ and the actual number of patches occupied by the larger relative $j$ for each $i-j$ combination once for day 15 (4 patches) and once for day 22 (8 patches). Expected versus observed patch sharing was then compared using nonparametric Wilxocon's tests. We performed the following additional analyses on pairs of relatives who had successfully settled (smaller focal $i$ vs. larger relative $j$ ). First, observed versus expected patch sharing was determined for pairs consisting of a subordinate and a dominant (subordinate $i$ vs. dominant $j$ or dominant $i$ vs. subordinate $j$ ). Second, observed versus expected patch sharing was determined for pairs consisting of 2 dominants. Third, we performed a linear stepwise regression through the origin with response variable (pairwise)-observed number of patches shared and as independent variables-expected number of patches shared (forcibly entered, i.e., always retained in the model), focal body size (SL in millimeters), difference in body size with relative (larger relative $j-$ focal relative $i$, SL in millimeters), focal sex (coded 1 for males or 0 for females), same sex as relative (coded 1 yes, 0 no), status obtained inside the ringtank on day 39 (coded 1 dominant, 0 subordinate), same status as relative (coded 1 yes, 0 no), and all their two-way interactions (using dummy variables) for 4 patches and 8 patches separately. None of the interactions were significant, and these were removed from the model presented.

\section{RESULTS}

\section{Experiment 1: body size, status, and reproduction}

The results of the first experiment show that dominant females and subordinate females from body size $32 \mathrm{~mm}$ SL upwards are reproductively capable (Figure 2). Clutch size (Figure 2a) and egg mass (Figure 2b, nonlinear) strongly increased with female body size (Table 2). Dominant females produced significantly more eggs for a given body size than subordinate

\section{Table 2}

Experiment 1. Female reproduction depending on female body size, status (dominant or subordinate), and number of subordinates $>25 \mathrm{~mm}$ SL inside their group. Results are shown of 2 general linear mixed model analyses, with individual identifier nested within status as random effect

\begin{tabular}{|c|c|c|c|c|c|}
\hline Parameters & df & Error df & $F$ & $P$ & Coefficient $\pm \mathrm{SE}$ \\
\hline Intercept & 1 & 82.7 & 82.9 & $<0.0001$ & $0.7517 \pm 0.1029$ \\
\hline Body size ${ }^{\mathrm{b}}$ & 1 & 73.1 & 108.8 & $<0.0001$ & $0.0164 \pm 0.0016$ \\
\hline Number of subordinates & 1 & 566.3 & 5.3 & 0.022 & $-0.0066 \pm 0.0029$ \\
\hline \multicolumn{6}{|c|}{ Average egg mass (mg, $n=545$ broods of 87 females $)^{\mathrm{d}}$} \\
\hline Intercept & 1 & 436.5 & 1.3 & 0.26 & $0.1009 \pm 0.0840$ \\
\hline Status $^{\mathrm{c}}$ & 1 & 100.6 & 0.6 & 0.46 & $-0.0142 \pm 0.0190$ \\
\hline Number of subordinates & 1 & 504.6 & 11.2 & 0.001 & $-0.0015 \pm 0.0004$ \\
\hline
\end{tabular}

\footnotetext{
${ }^{\mathrm{a}} \log 10$ transformed before analysis.
}

${ }^{\mathrm{b}}$ Standard length SL (millimeters).

${ }^{\mathrm{c}}$ Coefficient for dominant females, subordinate females have coefficient set to 0 .

d Twenty-nine of 545 dominant female and 2 of 31 subordinate female broods were consumed before egg mass could be determined, but the likelihood did not depend on female status (likelihood $\chi^{2}=0.07, \mathrm{df}=1, P=0.79$ ). 
Table 3

Experiment 2. Likelihood of acquiring group membership (dominant or subordinate) depending on their body size. Results are shown of a multinomial regression with aggregation as the reference category

\begin{tabular}{lcccr} 
Status acquired & df & Wald & $P$ & \multicolumn{1}{c}{$\begin{array}{l}\text { Coefficient } \\
\text { DE SE }\end{array}$} \\
\hline $\begin{array}{l}\text { Dominant } \\
\quad \text { Intercept }\end{array}$ & 1 & 50.4 & $<0.0001$ & $-28.896 \pm 4.070$ \\
$\quad \begin{array}{c}\text { Body size } \\
\text { Subordinate }\end{array}$ & 1 & 49.5 & $<0.0001$ & $0.532 \pm 0.076$ \\
$\quad \begin{array}{l}\text { Intercept } \\
\text { Body size }\end{array}$ & 1 & 3.2 & 0.072 & $2.685 \pm 1.491$ \\
& 1 & 8.5 & 0.004 & $-0.100 \pm 0.034$
\end{tabular}

Sample size is 20 trials with 5 females and 5 males each (of 5 different size classes). Sex $(P=0.48)$ and the interaction between sex $\times$ body size $(P=0.86)$ were nonsignificant and removed from the model. The final model shown has a pseudo- $R^{2}$ of 0.53 (Nagelkerke) and goodness-of-fit deviance $=62.7, \mathrm{df}=90, P=0.99$.

${ }^{\text {a }}$ Standard length SL (millimeters).

females (Figure 2a), but egg mass did not depend on social status (Figure 2b, Table 2). Clutch size and egg mass decreased with the number of subordinates present in the group for both types of females (all interactions between the main effects were nonsignificant, Table 2). The results suggest that small females are reproductively capable and should try to compete for dominant group membership (see experiment 2), settle independently when habitat is available (see experiment 3), and might need to avoid kin when targeting own reproduction (e.g., inbreeding avoidance, see experiments 3 and 4 ). Note that previous parentage studies have also shown that small males are reproductively capable (i.e., parasitize the reproduction of the dominant males, see references in INTRODUCTION), so similar arguments also apply to the small males as apply to the small females.

\section{Experiment 2: competition for breeding positions}

The results of the second experiment show that large females (Figure 2c) and males (Figure 2d) successfully competed for the dominant reproductive position, whereas small cichlids were accepted as subordinates and medium cichlids remained inside the aggregation (Figure $2 \mathrm{c}-\mathrm{d}$, Table 3 ). Dominants accepted on average 1.20 subordinates $( \pm 0.70$ standard error of the mean [SEM], range $0-2, n=20$ trials; $n=3 \times 0$ subordinate, $n=10 \times 1$ subordinate, and $n=7 \times 2$ subordinates were accepted). Subordinates were of a different sex in the 7 cases where 2 subordinates were accepted. The size difference between the dominants and same-sex subordinates was significantly larger (both for females, Figure 2e, and males, Figure 2f) than the size difference between the dominants and same-sex aggregation fish (ANOVA effect of same-sex status $F_{1,137}=5.5, P=0.021$; no effects of sex $F_{1,137}=1.5$, $P=0.23$, sex $\times$ status $F_{1,137}=0.07, P=0.79$, and trial $F_{1,137}=$ $0.86, P=0.64)$. The results suggest that small and medium cichlids may need to accept a subordinate position when the habitat gets saturated (see experiment 3 ).

\section{Experiment 3: habitat saturation}

The results of the third experiment show that both habitat saturation and competition among differently sized fish determine the extent of group living (Table 4). Cichlids appeared to notice the extent of saturation on release, as 1) the initial number of different breeding patches visited significantly declined through the experiment (GLM, $n=216)$ : day of release (Wald $\chi^{2}=12.6$, degrees of freedom $[\mathrm{df}]=1, P<$ $0.001, B=-0.024 \pm 0.007$ ) and size class (Wald $\chi^{2}=12.1$, $\mathrm{df}=2, P=0.002)$ were significant, and the effect of trial was nonsignificant (Wald $\chi^{2}=3.0, \mathrm{df}=2, P=0.22$ ). Small cichlids visited significantly more patches $(B=0)$ than large cichlids $(B=-0.619 \pm 0.179, P=0.001)$ but did not visit more patches than medium cichlids $(B=-0.253 \pm 0.160, P=$ $0.113)$. Moreover, 2) the number of patches visited are correlated significantly with the frequency of aggression received by the already established fish (Spearman rank correlation, $n=$ $\left.216, r_{\mathrm{s}}=0.78, P<0.001\right)$.

Co-operative breeding emerged directly from the start of each trial (Figure 3a-c: i.e., fish settled as subordinates are the light gray bars on days 3, 4 etc.), where large dominants accepted smaller-sized fish as subordinates (Figure 3i: e.g., all 18 small individuals released on day 0 settled as subordinates eventually). Due to habitat saturation, the number of fish not accepted as group members steadily increased through each trial, such that at the end of each trial only a few new fish settled inside the patches (Figure $3 a-c$ ). Both the number of dominant females (Figure 3d) and males (Figure 3e) gradually increased but leveled off at 15-17 females and 8-9 males at the end of each trial (Poisson Regression, effects of sex Wald $\chi^{2}=5.3$, df $=1, P=0.021$, days since start trial Wald $\chi^{2}=775.3, \mathrm{df}=1, P<0.0001$, sex $\times$ days Wald $\chi^{2}=11.8$, $\mathrm{df}=1, P=0.001$, trial Wald $\left.\chi^{2}=1.5, \mathrm{df}=2, P=0.47\right)$. The average number of subordinates accepted by each dominant female was $1.92( \pm 0.26$ SEM, range $0-6, n=48$, at day 39 ) and did not significantly increase during the experiment (Figure 3f, Poisson regression, effects of days since start trial Wald $\chi^{2}=0.15, \mathrm{df}=1, P=0.70$, trial Wald $\chi^{2}=0.35$, $\mathrm{df}=2$, $P=0.84)$.

\section{Table 4}

Experiment 3: final status achieved per trial depending on body size and sex. Depicted are totals in bold, with results for the separate trials 1, 2, and 3 in brackets. Total $n=216$ individuals

Sex, body

size

Aggregation

Subordinate

Dominant

Total

\begin{tabular}{lr}
\hline Males & \\
Large & $\mathbf{1 3}(3,4,6)$ \\
Medium & $\mathbf{1 1}(2,6,3)$ \\
Small & $\mathbf{4}(1,2,1)$ \\
Females & \\
Large & $\mathbf{5}(1,3,1)$ \\
Medium & $\mathbf{6}(1,3,2)$ \\
Small & $\mathbf{3}(1,0,2)$ \\
Total & $\mathbf{4 2}(9,18,15)$
\end{tabular}
$1(1,0,0)$
$21(10,2,9)$
$32(11,12,9)$
0 $(0,0,0)$
$17(8,5,4)$
$29(10,10,9)$
$100(40,29,31)$

$25(8,9,8)$

$1(0,0,1)$

$\mathbf{0}(0,0,0)$

$28(11,8,9)$

$16(3,8,5)$

$4(1,0,3)$

$74(23,25,26)$
$39(12,13,14)$ $33(12,8,13)$

$36(12,14,10)$

$33(12,11,10)$ $39(12,16,11)$

$36(12,10,14)$

$216(72,72,72)$ 


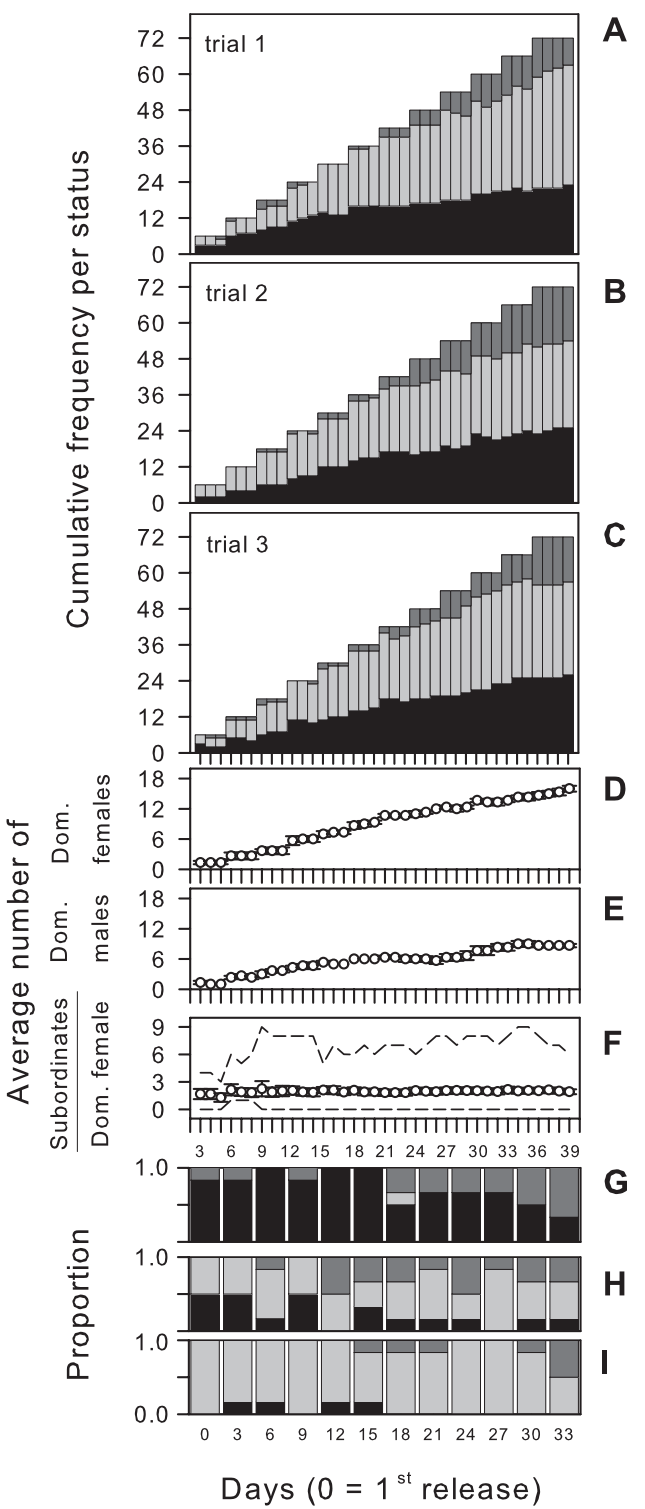

Figure 3

Experiment 3: habitat saturation and co-operative breeding. The number of individuals of dominant (black), subordinate (gray), and aggregation status (dark gray) during (a) trial 1, (b) trial 2, and (c) trial 3. The average number per trial and day of (d) dominant females and (e) dominant males $(n=3$ each dot). (f) The average number of subordinates per dominant female $(n=$ between 6 and 48 ), with the lower and upper dashed lines indicating minimum and maximum. The proportion of $(\mathrm{g})$ large, $(\mathrm{h})$ medium, and (i) small cichlids achieving dominant (black), subordinate (gray), or aggregation status (dark gray) at the end of the experiment (day 39) depending on the day of their release $(0-33, n=6$ each bar).

Body size and sex of the individuals cointeracted with habitat saturation to determine the extent of co-operative breeding in these cichlids (Tables 4 and 5): large fish mainly settled as dominants (Figure $3 \mathrm{~g}$ ), medium fish as dominants or subordinates (Figure 3h), and small fish as subordinates (Figure 3i), but all fishes of all size classes were more likely to end up in the aggregation as the experiment progressed (Figure $3 g-i$ ). Females were more likely to settle as dominants (because they could mate polygynously), also for a smaller size compared with males, and males of intermediate sizes were more likely to remain inside the aggregation, particularly when the habitat was already saturated (Figure 4, Table 5).

\section{Experiments 3 and 4: sequential and simultaneous settlement with kin}

To test the kin selection hypothesis, we also examined whether individuals preferred to settle with relatives in experiments 3 and 4 (relatives were full siblings, see Supplementary material). If cichlids go for kin-selected benefits, small and medium individuals should settle as subordinates with large relatives, that is, with large relatives who successfully settled as dominants. If cichlids go for direct own reproductive participation and avoid inbreeding, they should settle with nonrelatives.

In the third experiment, we tested this by releasing trios or duos of relatives sequentially on different days ("sets", e.g., large relative on day $i$, medium relative on day $i+3$, and small relative on day $i+6$ or vice versa). Then, we compared the pairwise settlement pattern of these fish that either settled as subordinates or dominants, discarding fish with no relatives settled at all. Within duos of settled relatives, subordinates and dominants occupied and defended between 1 and 4 patches of the 24 patches available $(1: n=38,2: n=17,3: n=3$, and 4 patches: $n=1$ ). However, only 2 of 29 small and 0 of 22 medium cichlids settled with a large relative, and 5 of 31 small cichlids settled with a medium relative (see Supplementary material Table S2 for the details). Using the hypergeometric distribution, we calculated the pairwise expected number of patches shared between 2 relatives under random settlement (24 patches) and compared these with the pairwise observed patches shared between two relatives (Figure 5a). Small and medium cichlids avoided settlement with related large individuals (Wilxocon's test $z=-5.3, n=51, P<0.001$ ), and small cichlids avoided settlement with related medium individuals $(z=-2.0, n=31, P=0.041)$. Only 1 small male and 1 small female subordinate settled with a related dominant female and might acquire kin-selected benefits from their helping behavior, whereas a small dominant female and a large dominant male were inbreeding (see Supplementary material Table S2 for the details). However, one might argue

\section{Table 5}

Experiment 3: habitat saturation, body size, sex, and final status achieved. Results are shown of a multinomial regression analysis, with the response variable status achieved (dominant, subordinate, or aggregation) depending on the day of release in the ringtank $(0,3$, $6,9 \ldots 33$ days), body size, sex, and trial $(1,2$, or 3$), n=216$ individuals

\begin{tabular}{|c|c|c|c|c|c|}
\hline Parameter & $\mathrm{df}$ & $\chi^{2}$ & $P$ & $\begin{array}{c}\text { Coefficient } \\
\stackrel{ \pm}{\text { SE subordinate }^{\mathrm{a}}}\end{array}$ & $\begin{array}{c}\text { Coefficient } \\
\pm \\
\text { SE dominant } \\
\text { a }^{\mathrm{a}}\end{array}$ \\
\hline Inter & & & & 8.565 & -9.199 \\
\hline Day released & 2 & 33.3 & $<0.0001$ & $-0.063 \pm 0.024$ & $-0.146=$ \\
\hline Body size ${ }^{b}$ & 2 & 182.1 & $<0.0001$ & $-0.175 \pm 0.033$ & $0.228 \pm 0.049$ \\
\hline $\operatorname{Sex}^{c}$ & 2 & 35.5 & $<0.0001$ & $0.255 \pm 0.480$ & $3.277 \pm 0.698$ \\
\hline \multicolumn{6}{|l|}{ Trial $^{\mathrm{d}}$} \\
\hline $\begin{array}{l}\text { Trial } 1 \\
\text { Trial } 2\end{array}$ & 4 & 8.0 & 0.09 & $\begin{array}{r}0.923 \pm 0.587 \\
-0.514 \pm 0.540\end{array}$ & $\begin{array}{l}-0.316 \pm 0.686 \\
-0.360 \pm 0.595\end{array}$ \\
\hline
\end{tabular}

a Aggregation is the reference category.

${ }^{\mathrm{b}}$ Standard length SL (millimeters).

${ }^{\mathrm{c}}$ Coefficients for females, males have coefficients set to 0 .

${ }^{\mathrm{d}}$ Coefficients for trials 1 (up) and 2 (below); trial 3 has coefficients set to 0 . 


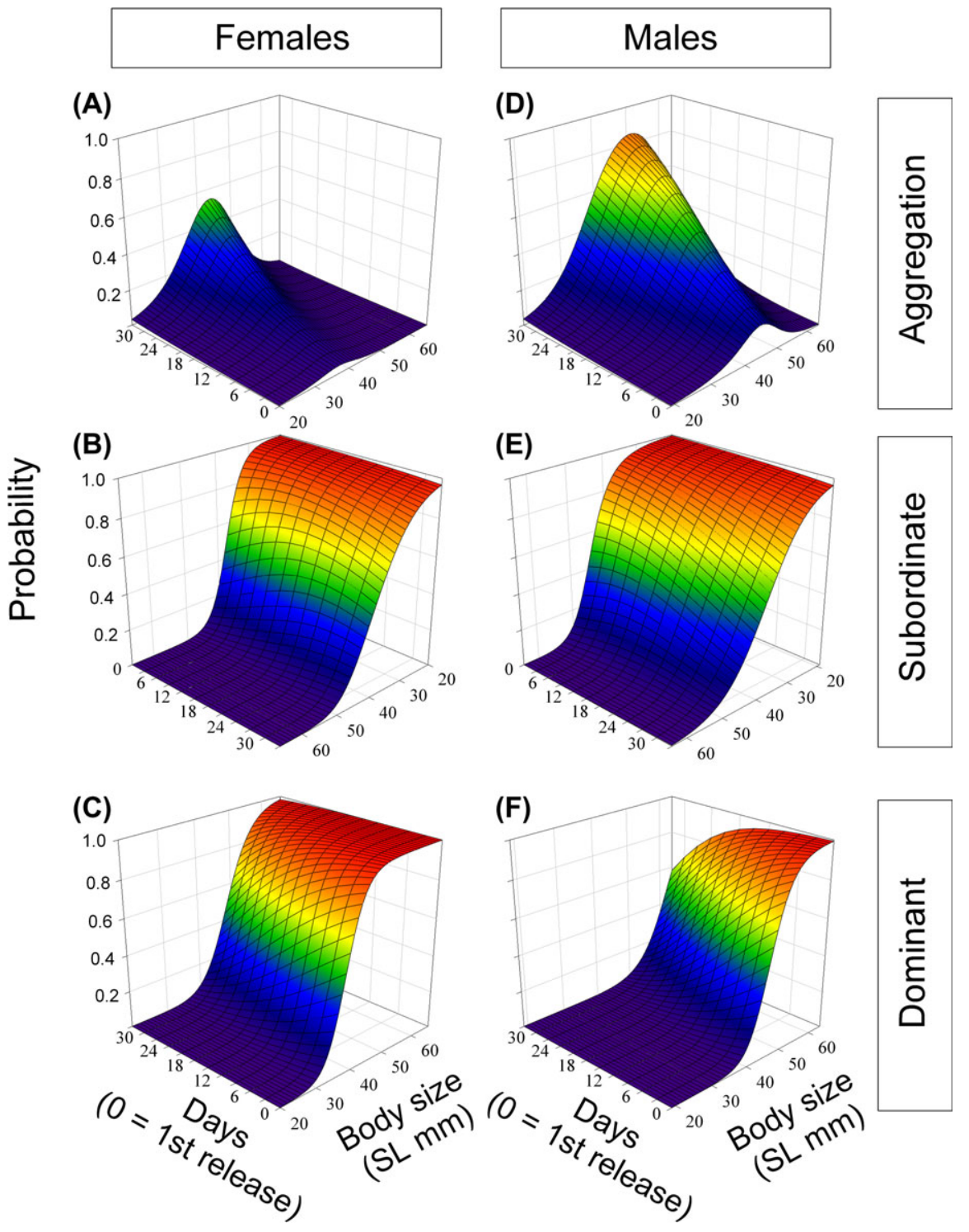

Figure 4

Experiment 3: habitat saturation, body size, and sex interactions. The likelihood of females $(a-c)$ and males $(d-f)$ achieving ( $a$ and d) aggregation ( $b$ and $e)$, subordinate, or (c and $\mathrm{f}$ ) dominant status at the end of the experiment (day 39), depending on their body size and day of release (model fits from Table 5). Note that for clarity, the "days" axis has been reversed for panels (b) and (e).

that due to the sequential release of relatives, cichlids had difficulties in finding a vacancy with a relative to settle in.

Therefore, we also conducted a fourth experiment, where 6 relatives of 4 families each ( 3 size classes $\times 2$ sexes per family) were released simultaneously inside the ringtank (day 0 ) with only 4 patches to settle in and measured settlement on day 15 . Then, we added another 4 patches and again measured the distribution over the patches on day 22 (8 patches). In total, over all trials, 111 of 144 individuals (4 patches available) and 141 of 144 individuals ( 8 patches available), had settled as a dominant or subordinate (the remainder did not settle and were "aggregation fish" staying near the water surface of the ringtank). Again, we calculated the expected and observed patch overlap for experiment 4 (separately for 4 or 8 patches available). Settled cichlids who had settled relatives inside the ringtank occupied and defended between 1 and 3 patches when 4 patches or 8 patches were available (1: $n=82$ or 100, 2: $n=3$ or 15, and 3: $n=2$ or 1 , for 4 or 8 patches separately). Again, pairwise settlement with related individuals was relatively rare: in 62 of 215 cases cichlids settled with relatives $(28.8 \%, 4$ patches available) or in 57 of 340 cases ( $16.8 \%$, with 8 patches available). Settlement was random in respect to the expected patch sharing when 4 patches were available (Figure 5b, Wilxocon's test $z=-1.1, n=215, P=$ $0.25)$ and cichlids avoided relatives when 8 patches were available (Figure $5 \mathrm{c}, z=-6.4, n=340, P<0.001$ ). We redid these analyses for pairs consisting of a subordinate and a dominant (subordinate $i$ vs. dominant $j$ or dominant $i$ vs. subordinate $j$ ). Settlement was random in respect to the expected patch sharing when 4 patches were available (Wilxocon's test $z=-0.3$, $n=113, P=0.75)$, and cichlids avoided relatives when 8 patches were available $(z=-3.4, n=187, P=0.001)$. Then we redid the analyses for pairs consisting of two dominants. Again, settlement was random in respect to the expected patch sharing when 4 patches were available (Wilxocon's test $z=-1.6, n=29, P=0.11)$, and cichlids avoided relatives when 8 patches were available $(z=-3.6, n=51, P<0.001)$. Finally, we performed a linear stepwise regression through the origin with response variable (pairwise): observed number of patches shared and as independent variables: expected number of patches shared (forcibly entered), focal body size (SL in millimeters), difference in body size with relative (larger relative $j$ - focal relative $i$, SL in millimeters), focal sex (coded 1 for males or 0 for females), same sex as relative (coded 1 yes, 

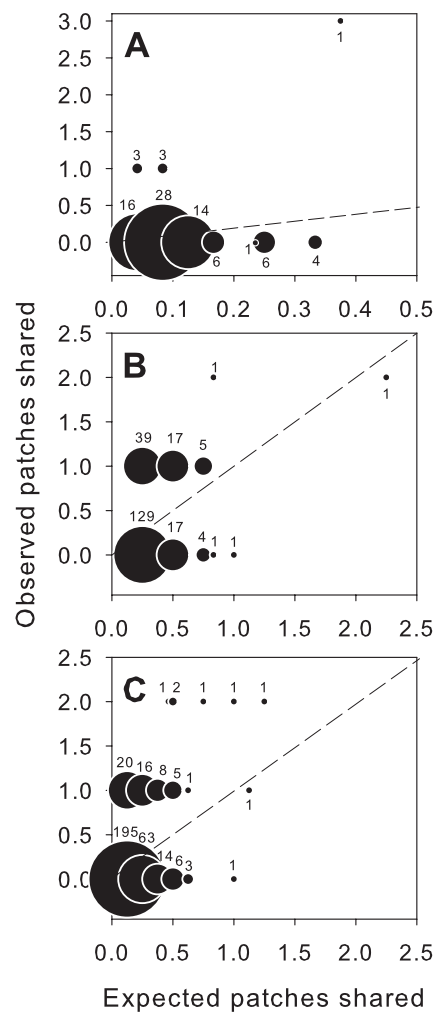

Figure 5

Experiments 3 and 4: effects of kinship on settlement in cichlids. Depicted are pairwise for 2 related individuals who both settled successfully: The observed number of patches shared with the related individual versus the expected number of patches shared with the related individual under random settlement. (a) Experiment 3 (24 patches available, $n=82$ pairwise comparisons); (b) experiment 4 ( 4 patches available, $n=215$ ), and (c) experiment 4 (8 patches available, $n=340$ ). Increasing symbol sizes denote increasing sample sizes, and the samples sizes are depicted alongside the symbols.

0 no), status ringtank (coded 1 dominant, 0 subordinate), same status as relative (coded 1 yes, 0 no), and all their twoway interactions (using dummy variables) for 4 patches and 8 patches separately (Table 6). None of the interactions were significant.
When 4 patches were available, the observed number of patches shared only significantly increased with the expected number of patches shared (Table 6 ). When 8 patches were available, the observed number of patches shared significantly increased with the expected number of patches shared and significantly decreased with the focal's body size (Table 6). Running the same analysis separately for subordinatedominant pairs revealed only a positive significant effect of the expected number of patches shared on the observed patch sharing (4 patches: $R^{2}=0.353, n=113, P<0.001$ and 8 patches: $\left.R^{2}=0.342, n=187, P<0.001\right)$. Running the same analysis separately for dominant-dominant pairs revealed only a significant effect of the expected number of patches shared on the observed patch sharing when 4 patches were available (4 patches: $R^{2}=0.530, n=29, P<0.001$ ), but an additional negative significant effect of body size SL when 8 patches were available $\left(R^{2}=0.513, n=51, P<0.001\right.$, effect of body size: $P=0.01$ with coefficient $-0.004 \pm 0.002 \mathrm{SE}$ ).

Taken together, these results strongly suggest that focal cichlids were mainly avoiding inbreeding or avoiding competition with kin, by avoiding settlement with relatives: avoidance of relatives increased with body size when more patches became available (i.e., when breeding became more likely and when avoidance was also more easy to achieve compared with only 4 patches available), particularly for pairs of related dominants of larger size (who are the most likely to participate in reproduction).

\section{DISCUSSION}

Our experiments show that although small cichlids are reproductively capable, they are excluded from breeding positions and accept a subordinate helper position instead, where they are unlikely to reproduce themselves (see also Heg et al. 2006; Heg 2008; Heg et al. 2008b). Habitat saturation determined the sizes and sexes of competitors accepted as group members and whether they achieved dominant status. However, habitat saturation appeared not necessary for cooperative breeding to emerge because small individuals also accepted a subordinate position when free habitat was still available, supporting previous results (Heg et al. 2008a). Large cichlids first settled as dominants but were then progressively excluded from groups, and males were less likely to settle than females, leading to polygyny (Limberger 1983). Medium cichlids sometimes settled as dominants, but these

\section{Table 6}

Experiment 4: linear regression through the origin of the observed number of patches shared depending on the expected patches shared under random settlement, parameters of the smaller focal $i$, and differences of these parameters with the larger relative $j$. Sample sizes are pairwise settlement patterns of a smaller focal $i$ and his/her larger relative $j$

\begin{tabular}{|c|c|c|c|c|}
\hline \multirow[b]{2}{*}{ Parameter } & \multicolumn{2}{|c|}{$\begin{array}{c}\text { Four patches available } \\
\text { (day } 15, n=215 \text { pairwise) }\end{array}$} & \multicolumn{2}{|c|}{$\begin{array}{l}\text { Eight patches available } \\
\text { (day } 22, n=340 \text { pairwise) }\end{array}$} \\
\hline & $B \pm \mathrm{SE}$ & $P$ & $B \pm \mathrm{SE}$ & $P$ \\
\hline Body size (SL mm) & & 0.90 & $-0.002 \pm 0.001$ & 0.009 \\
\hline Body size difference $(\Delta \mathrm{SL} \mathrm{mm})$ & & 0.23 & & 0.33 \\
\hline Sex (female or male) & & 0.39 & & 0.84 \\
\hline Status different ${ }^{\mathrm{a}}$ & & 0.85 & & 0.28 \\
\hline$R^{2}$ & 0.365 & & 0.334 & \\
\hline
\end{tabular}

Depicted are the coefficients $B \pm \mathrm{SE}$ and their significance $P$ of the two final models shown in bold with $R$-squared (and $P$-values of excluded variables when entered into these two final models).

${ }^{\mathrm{a}}$ Coded yes or no. 
were mainly females, and both sexes accepted subordinate positions. Again, due to habitat saturation, later released medium cichlids were excluded from the groups, and this effect was more severe in the males. Small cichlids were accepted as subordinates also after the habitat was well saturated and only ended in the aggregation at the very end of the experiments. Four small females achieved dominance status, whereas none of the small males achieved this. Our results provide additional support to the notion that body size may have important effects on dominance, reproduction, and growth in co-operatively breeding cichlids (e.g., Heg et al. 2004b; Heg and Hamilton 2008), as is also increasingly found in other taxa (e.g., Clutton-Brock et al. 2001; Gilchrist et al. 2004; Russell et al. 2004; Tibbetts and Dale 2004).

Finally, cichlids rather avoided, than preferred, to settle with relatives, if they had a choice. This indicates that kin selection is not a necessary condition for co-operation to emerge in these cichlids but rather that kin structure in cichlids (Stiver et al. 2004, 2005; Dierkes et al. 2005) emerges due to constraints on dispersal (Heg et al. 2004a, 2004b; Bergmüller et al. 2005; Heg and Hamilton 2008). Kin avoidance is a sensible strategy if cichlids target own direct reproduction, and this would explain why larger sized individuals avoided settlement with kin because larger group members are more likely to participate in reproduction (Heg et al. 2006, 2008b, 2009; Heg 2008; Stiver et al. 2009). However, field data do not show active inbreeding avoidance, at least not in the dominant pair (Stiver et al. 2008), and we also detected 1 case of inbreeding in a dominant pair in experiment 4 . Moreover, in a dual-choice situation where subordinates could associate through plexiglass with either a familiar group (probably includes related and unrelated individuals, see Dierkes et al. 2005) or an unfamiliar group, subordinates preferred to associate with the familiar group (Jordan et al. 2010), although actual settlement was not measured as in our experiments. Inbred offspring appear completely viable (Heg D., personal observation), suggesting that kin avoidance might actually not curb the inclusive fitness of subordinates and dominants engaging in inbreeding (e.g., Kokko and Ots 2006). Kin avoidance is also a sensible strategy if cichlids thereby avoid competing with kin over valuable resources (e.g., Perrin and Mazalov 2000; Perrin and Lehmann 2001). Important resources in $N$. pulcher are food, shelter, and mates. Kin competition for food and shelters is unlikely to apply in $N$. pulcher, where zooplankton food floats freely through the territories and is also obtained by swimming away from the territories, and hiding shelters can be enlarged to fit all the group members. However, competition for mates may favor avoidance of kin, if mature group members target own reproduction.

Our habitat saturation experiment corroborates previous experimental work indicating that population density may affect sociality in vertebrates (Komdeur 1992; Harris et al. 1995; Lucia et al. 2008). In the only similar experiment to date, conducted in the Seychelles warblers Acrocephalus sechellensis, natal territory quality and saturation together determined the dispersal decisions of subordinates and therefore the extent of co-operative breeding (Komdeur 1992; Komdeur 1993; Komdeur and Edelaar 2001; Ridley et al. 2003). We showed that small cichlids prefer co-operative breeding irrespective of saturation (see Figure $3 \mathrm{a}-\mathrm{c}, \mathrm{i}$ ), also under laboratory conditions without predation risk (which is known to affect dispersal in the field: Heg et al. 2004a). However, habitat saturation did lead to progressively less individuals settling, and this effect was stronger for males and stronger for the intermediate (medium) size classes. The competition experiment suggests that this was due to dominant individuals (mostly large fish) only accepting subordinate individuals substantially smaller than themselves (i.e., mostly small fish).
Our results support the viewpoint (Koenig and Dickinson 2004) and theoretical work (Pen and Weissing 2000; Le Galliard et al. 2005) indicating that a life-history approach is needed to integrate all environmental and social factors affecting the individual's decision at each life stage whether to disperse or stay. We have shown that measurements of the fitness benefits/costs ratio of philopatry versus dispersal during the entire lifetime are necessary to understand settlement and dispersal decisions in co-operatively breeding vertebrates. We have also shown that kin structure appears to be an emergent property of cichlid societies due to limits on dispersal and settlement because in the absence of such limits, cichlids prefer to settle with nonrelatives.

\section{SUPPLEMENTARY MATERIAL}

Supplementary material can be found at http://www.beheco .oxfordjournals.org/.

\section{FUNDING}

Swiss National Science Foundation (SNSF 3100A-108473) to D.H.

We thank Eva Jutzeler and the Hasli members for their support and the referees for comments on the manuscript. We thank Sander van Doorn for his assistance with the hypergeometric distributions. The experiments described in this paper were approved by LANAT of the Kanton Bern, license number 40/05.

\section{REFERENCES}

Baglione V, Marcos JM, Canestrari D, Griesser M, Andreotti G, Bardini C, Bogliani G. 2005. Does year-round territoriality rather than habitat saturation explain delayed natal dispersal and cooperative breeding in the carrion crow? J Anim Ecol. 74:842-851.

Balshine-Earn S, Lotem A. 1998. Individual recognition in a cooperatively breeding cichlid: evidence from video playback experiments. Behaviour. 135:369-386.

Balshine S, Leach B, Neat F, Reid H, Taborsky M, Werner N. 2001. Correlates of group size in a cooperatively breeding cichlid fish (Neolamprologus pulcher). Behav Ecol Sociobiol. 50:134-140.

Balshine-Earn S, Neat FC, Reid H, Taborsky M. 1998. Paying to stay or paying to breed? Field evidence for direct benefits of helping behavior in a cooperatively breeding fish. Behav Ecol. 9:432-438.

Bergmüller R, Heg D, Taborsky M. 2005. Helpers in a cooperatively breeding cichlid stay and pay or disperse and breed, depending on ecological constraints. Proc R Soc Lond B Biol Sci. 272: $325-331$

Bull NJ, Schwarz MP. 1996. The habitat saturation hypothesis and sociality in an allodapine bee: cooperative nesting is not 'making the best of a bad situation'. Behav Ecol Sociobiol. 39:267-274.

Cahan SH, Blumstein DT, Sundström L, Liebig J, Griffin A. 2002. Social trajectories and the evolution of social behavior. Oikos. 96:206-216.

Clutton-Brock TH, Russell AF, Sharpe LL, Brotherton PNM, McIlrath GM, White S, Cameron EZ. 2001. Effects of helpers on juvenile development and survival in meerkats. Science. 293: 2446-2449.

Covas R, Griesser M. 2007. Life history and the evolution of family living in birds. Proc R Soc Lond B Bio Sci. 274:1349-1357.

Dierkes P, Heg D, Taborsky M, Skubic E, Achmann R. 2005. Genetic relatedness in groups is sex-specific and declines with age of helpers in a cooperatively breeding cichlid. Ecol Lett. 8:968-975.

Emlen ST. 1982. The evolution of helping. I. An ecological constraints model. Am Nat. 119:29-39.

Fitzpatrick JL, Desjardins JK, Stiver KA, Montgomerie R, Balshine S. 2006. Male reproductive suppression in the cooperatively breeding fish Neolamprologus pulcher. Behav Ecol. 17:25-33. 
Gilchrist JS, Otali E, Mwanguhya F. 2004. Why breed communally? Factors affecting fecundity in a communal breeding mammal: the banded mongoose (Mungos mungo. Behav Ecol Sociobiol. 57: $119-131$.

Grafen A. 2007. Detecting kin selection at work using inclusive fitness. Proc R Soc Lond B Bio Sci. 274:713-719.

Griesser M, Nystrand M, Ekman J. 2006. Reduced mortality selects for family cohesion in a social species. Proc R Soc Lond B Bio Sci. 273:1881-1886.

Hamilton IM, Heg D. 2008. Sex differences in the effect of social status on the growth of subordinates in a cooperatively breeding cichlid. J Fish Biol. 72:1079-1088.

Harris RN, Hames WW, Knight IT, Carreno CA, Vess TJ. 1995. An experimental analysis of joint nesting in the salamander Hemidactylium scutatum (Caudata, Plethodontidae): the effects of population density. Anim Behav. 50:1309-1316.

Heg D. 2008. Reproductive suppression in female cooperatively breeding cichlids. Biol Lett. 4:606-609.

Heg D, Bachar Z, Brouwer L, Taborsky M. 2004a. Predation risk is an ecological constraint for helper dispersal in a cooperatively breeding cichlid. Proc R Soc Lond B Bio Sci. 271:2367-2374.

Heg D, Bender N, Hamilton I. 2004b. Strategic growth decisions in helper cichlids. Proc R Soc Lond B Bio Sci. 271:S505-S508.

Heg D, Bergmüller R, Bonfils D, Otti O, Bachar Z, Burri R, Heckel G, Taborsky M. 2006. Cichlids do not adjust reproductive skew to the availability of independent breeding options. Behav Ecol. 17:419-429.

Heg D, Brouwer L, Bachar Z, Taborsky M. 2005. Large group size yields group stability in the cooperatively breeding cichlid Neolamprologus pulcher. Behaviour. 142:1615-1641.

Heg D, Hamilton IM. 2008. Tug-of-war over reproduction in a cooperatively breeding cichlid. Behav Ecol Sociobiol. 62:1249-1257.

Heg D, Heg-Bachar Z, Brouwer L, Taborsky M. 2008a. Experimentally induced helper dispersal in colonially breeding cooperative cichlids. Environm Biol Fish. 83:191-206.

Heg D, Jutzeler E, Bonfils D, Mitchell JS. 2008b. Group composition affects male reproductive partitioning in a cooperatively breeding cichlid. Mol Ecol. 17:4359-4370.

Heg D, Jutzeler E, Mitchell JS, Hamilton IM. 2009. Helpful female subordinate cichlids are more likely to reproduce. PLoS ONE. 4: e5458.

Hert E. 1985. Individual recognition of helpers by the breeders in the cichlid fish Lamprologus brichardi (Poll, 1974). Z Tierpsychol. 68: 313-325.

Jordan LA, Wong MYL, Balshine S. 2010. The effects of familiarity and social hierarchy on group membership decisions in a social fish. Biol Lett. 6:301-303.

Koenig WD, Dickinson JL. 2004. Ecology and evolution of cooperative breeding in birds. Cambridge: Cambridge University Press.

Kokko H, Ots I. 2006. When not to avoid inbreeding. Evolution. 60:467-475.

Komdeur J. 1992. Importance of habitat saturation and territory quality for evolution of cooperative breeding in the seychelles warbler. Nature. 358:493-495.

Komdeur J. 1993. Fitness-related dispersal. Nature. 366:23-24.

Komdeur J, Edelaar P. 2001. Male seychelles warblers use territory budding to maximize lifetime fitness in a saturated environment. Behav Ecol. 12:706-715.

Langer P, Hogendoorn K, Keller L. 2004. Tug-of-war over reproduction in a social bee. Nature. 428:844-847.

Le Galliard JF, Ferriere R, Dieckmann U. 2005. Adaptive evolution of social traits: origin, trajectories, and correlations of altruism and mobility. Am Nat. 165:206-224.

Limberger D. 1983. Pairs and harems in a cichlid fish, Lamprologus brichardi. Z Tierpsychol. 62:115-144.

Lucia KE, Keane B, Hayes LD, Lin YK, Schaefer RL, Solomon NG. 2008. Philopatry in prairie voles: an evaluation of the habitat saturation hypothesis. Behav Ecol. 19:774-783.
Macedo RH, Bianchi CA. 1997. Communal breeding in tropical guira cuckoos Guira guira: sociality in the absence of a saturated habitat. J Avian Biol. 28:207-215.

Norusis MJ. 2007. SPSS 15.0 Advanced statistical procedures companion. Upper Sadle River (NJ): Prentice Hall.

Pen I, Weissing FJ. 2000. Towards a unified theory of cooperative breeding: the role of ecology and life history re-examined. Proc R Soc Lond B Bio Sci. 267:2411-2418.

Perrin N, Mazalov V. 2000. Local competition, inbreeding, and the evolution of sex-biased dispersal. Am Nat. 155:116-127.

Perrin N, Lehmann L. 2001. Is sociality driven by the costs of dispersal or the benefits of philopatry? A role for kin-discrimination mechanisms. Am Nat. 158:471-483.

Ridley AR, Raihani NJ. 2007. Variable postfledging care in a cooperative bird: causes and consequences. Behav Ecol. 18:994-1000.

Ridley J, Komdeur J, Sutherland WJ. 2003. Population regulation in group-living birds: predictive models of the seychelles warbler. J Anim Ecol. 72:588-598.

Russell AF, Carlson AA, McIlrath GM, Jordan NR, Clutton-Brock T. 2004. Adaptive size modification by dominant female meerkats. Evolution. 58:1600-1607.

Selander RK. 1964. Speciation in wrens of the genus Campylorhynchus. Univ Calif Publ Zool. 74:1-224.

Skubic E, Taborsky M, McNamara JM, Houston AI. 2004. When to parasitize? A dynamic optimization model of reproductive strategies in a cooperative breeder. J Theor Biol. 227:487-501.

Stacey PB, Ligon JD. 1991. The benefits-of-philopatry hypothesis for the evolution of cooperative breeding-variation in territory quality and group size effects. Am Nat. 137:831-846.

Stiver KA, Desjardins JK, Fitzpatrick JL, Neff B, Quinn JS, Balshine S. 2007. Evidence for size and sex-specific dispersal in a cooperatively breeding cichlid fish. Mol Ecol. 16:2974-2984.

Stiver KA, Dierkes P, Taborsky M, Balshine S. 2004. Dispersal patterns and status change in a co-operatively breeding cichlid Neolamprologus pulcher: evidence from microsatellite analyses and behavioural observations. J Fish Biol. 65:91-105.

Stiver KA, Dierkes P, Taborsky M, Gibbs HL, Balshine S. 2005. Relatedness and helping in fish: examining the theoretical predictions. Proc R Soc Lond B Bio Sci. 272:1593-1599.

Stiver KA, Fitzpatrick J, Desjardins JK, Balshine S. 2006. Sex differences in rates of territory joining and inheritance in a cooperatively breeding cichlid fish. Anim Behav. 71:449-456.

Stiver KA, Fitzpatrick J, Desjardins JK, Neff B, Quinn JS, Balshine S. 2008. The role of genetic relatedness among social mates in a cooperative breeder. Behav Ecol. 19:816-823.

Stiver KA, Fitzpatrick J, Desjardins JK, Balshine S. 2009. Mixed parentage in Neolamprologus pulcher groups. J Fish Biol. 74:1129-1135.

Taborsky B, Skubic E, Bruintjes R. 2007. Mothers adjust egg size to helper number in a cooperatively breeding cichlid. Behav Ecol. 18:652-657.

Taborsky M. 1984. Broodcare helpers in the cichlid fish Lamprologus brichardi: their costs and benefits. Anim Behav. 32:1236-1252.

Tibbetts EA, Reeve HK. 2003. Benefits of foundress associations in the paper wasp Polistes dominulus: increased productivity and survival, but no assurance of fitness returns. Behav Ecol. 14: 510-514.

Tibbetts EA, Dale J. 2004. A socially enforced signal of quality in a paper wasp. Nature. 432:218-222.

van Vuren D, Armitage KB. 1994. Survival of dispersing and philopatric yellow-bellied marmots: what is the cost of dispersal? Oikos. 69:179-181.

Walters JR, Copeyon CK, Carter LH. 1992. Test of the ecological basis of cooperative breeding in red-cockaded woodpeckers. Auk. 109: $90-97$. 